\title{
Supporting Patient-Provider Communication and Engagement with Personal Informatics Data
}

\author{
Chia-Fang Chung \\ Human Centered Design \& Engineering \\ DUB Group, University of Washington \\ cfchung@uw.edu
}

\begin{abstract}
Personal informatics data are prevalent in clinical care today. My research builds an understanding patient and provider needs and challenges to advance the design of personal informatics systems and theoretical understandings of patient-provider collaboration. Through studies of patients with Irritable Bowel Syndrome and people who want to eat healthier, I study people's current tracking experiences and their collaborative review practices with providers. Based on these findings, I propose design and theoretical considerations regarding interactions among providers, patients, and patient-tracked data. I design and evaluate systems in situ to understand potential use of lightweight data collection and integration to support collaboration around personal informatics data.
\end{abstract}

\section{Author Keywords \\ Personal informatics; Patient-Provider communication; Chronic disease management; Self-tracking; Health; Wellness}

\section{ACM Classification Keywords}

H.5.m. Information interfaces and presentation (e.g., $\mathrm{HCI}$ ): Miscellaneous;

\section{INTRODUCTION}

The prevalence of self-tracking applications and wearable sensing devices create an opportunity for individuals to collect continuous, objective, and precise everyday health and lifestyle data to complement standard clinical measurements. However, many self-trackers who shared their data with health providers reported frustration with their providers not engaging with this data $[2,8]$. Understanding current experience, challenges, and design opportunities can help HCI, CSCW, and UbiComp

Permission to make digital or hard copies of all or part of this work for personal or classroom use is granted without fee provided that copies are not made or distributed for profit or commercial advantage and that copies bear this notice and the full citation on the first page. Copyrights for components of this work owned by others than ACM must be honored. Abstracting with credit is permitted. To copy otherwise, or republish, to post on servers or to redistribute to lists, requires prior specific permission and or a fee. Request permissions from Permissions@acm.org.

UbiComp/ISWC'17 Adjunct, September 11-15, 2017, Maui, HI, USA

(C) 2017 Association for Computing Machinery.

ACM ISBN 978-1-4503-5190-4/17/09..\$15.00

https://doi.org/10.1145/3123024.3123197 communities better support patient-provider collaboration using personal informatics data.

My research focuses on two populations who use personal informatics data to support everyday decisions: Irritable Bowel Syndrome (IBS) patients and people with healthy eating goals. IBS providers and patients commonly use journals to identify and manage personal triggers, while people with healthy eating goals frequently use diaries to monitor lifestyle changes and their influence on health indicators, such as weight. My research makes four contributions:

1. Understand patient and provider goals, experiences, and challenges of using personal informatics data for clinical care.

2. Develop design principles and theoretical considerations to support patient-provider collaboration of using personal informatics data.

3. Understand potential use and nonuse of lightweight data collection and integration for collaborative use of personal informatics data.

4. Develop design considerations to collect and integrate minimal contextual information to support collaborative review of personal informatics data.

\section{PATIENT AND PROVIDER GOALS}

To examine current use of personal informatics data in the clinic, we conducted a series of studies with providers and patients with IBS and weight management concerns. Through interviews with 21 providers [3], surveys with 211 patients, and interviews with 18 patients [4], we found that both providers and patients want to use personal informatics data to support diagnosis and to provide personalized treatment. Reviewing this data together in the clinic can build rapport by surfacing a more complete view of patient life, values, and priorities and by increasing patient motivation and accountability.

Current systems and workflows often do not support the communication and implementation of these goals. Providers do not have enough time to review patienttracked data in detail. They were also unsure who in the medical team could best incorporate the data review process in their workflow. Current systems also do not provide the flexibility for providers and patients to focus on data most relevant for their needs and goals. 


\section{COLLABORATION USING PERSONAL INFORMATICS DATA}

Despite the challenges of integrating personal informatics data into clinic visit, many providers and patients currently use this data collaboratively. Using the stage-based model of personal informatics [10] to analyze our data, we found that providers and patients collaborate during every stage of tracking (Textbox 1). This personal informatics data was used as or transformed into various boundary negotiating artifacts [9] to support patient-provider collaboration. Transforming or moving this data through healthcare contexts, creates patient privacy tensions that are often not adequately addressed (Textbox 2) [4]. Providers and patients need better support to communicate and implement their collaborative review goals, to track and review data relevant to these goals, and to address related privacy concerns.

\section{DESIGN AND EVALUATION OF SYSTEMS}

Among all the lifestyle data, food is one of the most difficult type of data to track and analyze. Collecting food intake using traditional food journals (Figure 1, left and middle) requires knowledge of nutrient content and portion. Hence it often negatively nudges people to eat foods that are easy to track, such as packaged food [6].

Nevertheless, providers commonly recommend food journals to identify and manage individualized IBS triggers [7] and to understand eating behavior to achieve healthy eating goals [12]. However, in a study with eight IBS providers reviewing 17 food and symptom journals [13], we found all providers offered different clinical recommendations to the same patient. Individual providers also offered the same recommendations for multiple patients, regardless of what their diaries showed. These findings suggest that providers need better tools and methods for detailed review.

One approach for reducing burdens of patient data collection and provider review might be to use photo-based food journals. Photo-based food journaling can be less burdensome in terms of time and social appropriateness, and it still supports assessment of progress and failures [2,5]. Reviewing photos can help people remember the context around eating [2,5], which might in turn support patient-provider communication over everyday decisions and personal values that influence eating choices.

Photo-based food journaling, however, captures less detail than paper-based diaries. I am currently conducting a deployment study to examine the tradeoffs between these two different types of food data analysis - photo-based (Figure 1, right) and nutrient-based visualizations [11] and their use in collaborative review. Based on the design guidelines we learnt from prior survey and interview studies, I designed a photo-based food diary app and visualized summaries to support IBS patients and people with healthy eating goals sharing and reviewing their data with health providers. Our goal is to understand how and
TEXTBOX 1: Patients and providers collaborate during all stages of tracking [4]

Preparation: Patients make tracking decisions based on collaborative review goals. However, patients might not know what tools to use or what to track to better support these goals.

Collection: Provider review increases patient motivation and accountability. However, patients do not know what to expect from providers.

Integration: Patients want to curate data before sharing with providers. Current systems often do not support individualized, specific curation.

Reflection: Providers and patients attempt to review data together, but typically have less than five minutes per visit to do so.

Action: Providers make clinical recommendations and instructions, but patients do not know how to incorporate these into their tracking tools.

TEXTBOX 2: Personal informatics data are transformed into Boundary Negotiating Artifacts [4]

Self-explanation artifacts: Personal informatics data were first collected for personal use, without knowledge about who will review the data.

Inclusion artifacts: Patients and providers discuss whether and how to include these data into medical decisions. However, current tools often do not allow patients to easily choose what to share, and patients often rely on providers to make these decisions.

Compilation artifacts: Personal informatics data are input into the medical record. However, patients have no control over who can see these data beyond the current clinic encounter.

Structuring artifacts: Providers deliver handouts or instructions for patients to follow at home

when photo-based diaries help support people self-manage their symptoms, lifestyle, and health goals as well as communicate this information with health experts. We deploy the photo-based food diary app to IBS patients and people with health eating goals to understand how the lowburden data collection can support specific health goals and self-management. We then invite patients and providers back to review patient-tracked data separately and together to understand how and when these visualized food and symptom/lifestyle summaries can support individual and collaborative review. By comparing the use of photo-based visualizations with nutrient-based visualizations [11], we hope to understand when and how each visualization supports people working to manage IBS (particularly for 

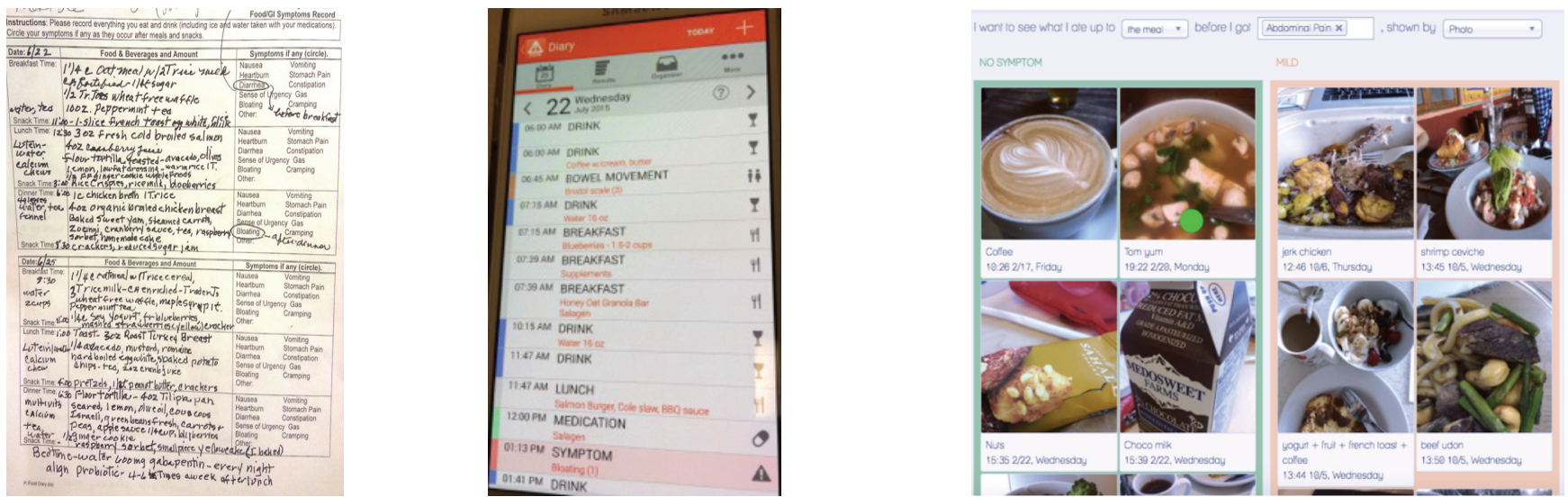

Figure 1. Traditional food diaries and photo-based diary we are deploying. Left: a paper-based diary. Middle: a mobile app diary. Right: Photo-based visualization summary we are deploying.

trigger identification) and to make healthier eating choices. Through these studies, we also seek to learn what information - the manually journaled or automatically sensed - can best complement photos.

\section{COLLECTING AND INTEGRATING RELEVANT CONTEXT TO SUPPORT COLLABORATIVE REVIEW}

Understanding cause and effect relationships and opportunities to tune behavior can also require integration of some contextual information such as location, social events, or weather. Although mobile phones and sensing devices are available to automatically collect potential relevant contextual information, too much information and complex data integration remain barriers for providers and patients [1].

In my prior work, we found that which contextual information is important and relevant depends on both specific health challenges as well as provider and patient goals for sharing and reviewing personal informatics data $[3,4,13]$. Traditionally, providers ask patients to verbally summarize this information during clinic visits. However, prior studies show that self-trackers often do not track the right context [1]. To understand the value of contextual information and the process of gathering it to help providers and patients make sense of personal informatics data individually and collaboratively, I plan to conduct further interviews and observations with providers who have experience reviewing personal informatics data. I plan to use design probes to understand how systems can support providers and patients to collect and integrate relevant contextual information into their collaborative review process. Based on the findings from the photo-based diaries, I also plan to iterate designs and conduct small scale deployment to understand how these designs can support personal informatics data collection, integration, and review in the context of patient-provider collaboration.

\section{CONTRIBUTION TO UBIQUITOUS COMPUTING}

My dissertation work contributes to the UbiComp community by bridging the gap between technology initiatives - self-monitoring devices, apps and data integration frameworks - and health improvement that relies on understanding patient-provider practices, workflows, and interactions. My research contributes novel system designs and workflows, improves theoretical models of patient-provider communication, and builds our understanding of how people with different expertise collaborate.

I have completed a series of studies understanding current use of personal informatics data in medical care and designs to support this use and published in JMIR [3], CSCW 2016 [4], and CSCW 2017 [11]. I have also studied how people appropriate Instagram for food journaling and published in CHI 2017 [2]. These papers offer an in-depth understanding about current practice and experience of using personal informatics data in healthcare as well as design and theoretical considerations. Based on this understanding, I am currently deploying a mobile app and its companion web app to support providers and patients with IBS in collecting and reviewing patient-tracked data in the University of Washington health system. I am also extending the system design to people with healthy eating goals and plan to deploy the second system in summer 2017. I plan to follow these up with research on integration of additional contextual information in collaborative review. I expect the results of these studies will contribute both novel system design to support integrating and reflecting personal informatics data individually and collaboratively as well as an understanding of experience and use in situ.

\section{UBICOMP 2017 DOCTORAL COLLOQUIUM}

I will complete my dissertation proposal in summer 2017 and complete my PhD in spring 2018. The timing of the UbiComp 2017 doctoral colloquium will give me the opportunity to receive feedback on preliminary results, analysis, and career trajectory from mentors and a strong cohort of peers. I have published primarily in medical journals, CSCW, and CHI, yet I see ubiquitous sensing and computing as essential components in identifying and addressing many chronic health conditions, and I expect 
that UbiComp will be an important community for me in the future. I hope that participating in the UbiComp doctoral consortium can help orient me and my research to the UbiComp community.

\section{ACKNOWLEDGMENTS}

My work was funded by the Intel Science and Technology Center for Pervasive Computing, AHRQ \#1R21HS023654, and NSF\#s OAI-1028195 and IIS-1344613.

\section{REFERENCES}

1. Eun Kyoung Choe, Nicole B. Lee, Bongshin Lee, Wanda Pratt, and Julie A. Kientz. 2014. Understanding quantified-selfers' practices in collecting and exploring personal data. In Proceedings of the SIGCHI Conference on Human Factors in Computing Systems (CHI 2014). ACM, New York, NY, USA, 1143-1152. http://doi.org/10.1145/2556288.2557372

2. Chia-Fang Chung, Elena Agapie, Jessica Schroeder, Sonali Mishra, James Fogarty, Sean A Munson. 2017. When Personal Tracking Becomes Social: Examining the Use of Instagram for Healthy Eating. In Proceedings of the SIGCHI Conference on Human Factors in Computing Systems (CHI 2017). ACM, New York, NY, USA, 1143-1152, 1674-1687. https://doi.org/10.1145/3025453.3025747

3. Chia-Fang Chung, Jonathan Cook, Elizabeth Bales, Jasmine Zia, and Sean A. Munson. 2015. More Than Telemonitoring: Health Provider Use and Non-Use of Lifelog Data in Irritable Bowel Syndrome and Weight Management. J Med Internet Res; 17(8):e203. http://doi.org/10.2196/jmir.4364

4. Chia-Fang Chung, Kristin Dew, Allison Cole, Jasmine K. Zia, James Fogarty, Julie A. Kientz, and Sean A. Munson. 2016. Boundary Negotiating Artifacts in Personal Informatics: Patient-Provider Collaboration with Patient-Generated Data. In Proceedings of the ACM conference on Computer-Supported Cooperative Work and Social Computing (CSCW 2016), 770-786. http://doi.org/10.1145/2818048.2819926

5. Felicia Cordeiro, Elizabeth Bales, Erin Cherry, and James Fogarty. 2015. Rethinking the Mobile Food Journal. Proceedings of the SIGCHI Conference on Human Factors in Computing Systems (CHI 2015): 3207-3216. http://doi.org/10.1145/2702123.2702154

6. Felicia Cordeiro, Daniel A Epstein, Edison Thomaz, Cordeiro, Elizabeth Bales, Arvind K Jagannathan, Gregory D Abowd, and James Fogarty. 2015. Barriers and Negative Nudges: Exploring Challenges in Food
Journaling. In Proceedings of the SIGCHI Conference on Human Factors in Computing Systems (CHI 2015): 1159-1162. http://doi.org/10.1145/2702123.2702155.

7. Gastroenterology. 2002. American Gastroenterological Association medical position statement: irritable bowel syndrome. 2002;123(6):2105-7.

8. Health Data Exploration Project. 2014. Personal Data for the Public Good: New Opportunities to Enrich Understanding of Individual and Population Health. Robert Wood Johnson Foundation.

9. Charlotte P. Lee. 2007. Boundary Negotiating Artifacts: Unbinding the Routine of Boundary Objects and Embracing Chaos in Collaborative Work. Comput. Supported Coop. Work; 16(3): 307-339. http://doi.org/10.1007/s10606-007-9044-5

10. Ian Li, Anind K. Dey and Jodi Forlizzi. 2010. A stagebased model of personal informatics systems. In Proceedings of the SIGCHI Conference on Human Factors in Computing Systems (CHI 2010). ACM, New York, NY, USA, 557-566. http://doi.org/10.1145/1753326.1753409

11. Jessica Schroeder, Jane Hoffswell, Chia-Fang Chung, James Fogarty, Sean A Munson, Jasmine K Zia. 2017. Supporting Patient-Provider Collaboration to Identify Individual Triggers using Food and Symptom Journals. In Proceedings of the ACM conference on ComputerSupported Cooperative Work and Social Computing (CSCW 2017), 1726-1739.

https://doi.org/10.1145/2998181.2998276

12. U.S. Preventive Services Task Force. 2012. Screening for and Management of Obesity in Adults. $A H R Q$ Publication No. 11-05159-EF-2. June 2012.

13. Jasmine K Zia, Chia-Fang Chung, Kaiyuan Xu, Yi Dong, Kevin C Cain, Sean A Munson, Margaret Heitkemper. 2016. Inter-rater Reliability of Healthcare Provider Interpretations of Food and Gastrointestinal Symptom Paper Diaries of Patients with Irritable Bowel Syndrome. 2016 Digestive Disease Week, San Diego, CA. 